\title{
Selected components of physical fitness in rhythmic and artistic youth gymnast
}

\author{
Luca Russo $^{1}$ (1) Stefano Palermi ${ }^{2} \cdot$ Wissem Dhahbi $^{3,7} \cdot$ Sunčica Delaš Kalinski ${ }^{4} \cdot$ Nicola Luigi Bragazzi $^{5}$. \\ Johnny Padulo ${ }^{6}$
}

Received: 9 April 2020 / Accepted: 24 October 2020 / Published online: 1 December 2020

(c) The Author(s) 2020

\begin{abstract}
Purpose Rhythmic (RG) and artistic gymnastics (AG) are very popular female sports. These two disciplines share some common points but, at the same time, they display some relevant differences in terms of physical and technical characteristics. The aim of this study was as follows: (1) to clarify how gymnastic training background over the years could lead to the development and motor learning of the motor skills and (2) to highlight differences of conditional skills achieved by RG and AG athletes.

Methods For these aims, 45 athletes were selected, belonging to three balanced groups: promotional (PG, $n=15$ ), RG $(n=15)$, and AG $(n=15)$. Participants were tested for joints mobility, balance, explosive strength, speed, and endurance tests. Results Statistical analysis showed a good test-retest reliability of the measurements (ICC $>0.870$ ) and some significant differences between PG, RG, and AG. RG showed higher values in joint mobility tests (coxo-femoral mobility, $166.7 \pm 6.3^{\circ}$; sit and reach, $20.5 \pm 1.9 \mathrm{~cm}$; and scapulo-humeral mobility, $45.5 \pm 4.4 \mathrm{~cm}$ ) with respect to AG, while AG showed higher values in endurance $(1626.7 \pm 7.4 \mathrm{~m})$, balance $(4.33 \pm 1.35 \mathrm{n} / 60 \mathrm{~s})$, and explosive strength $(164.1 \pm 11.6 \mathrm{~cm})$ compared to $\mathrm{RG}(p<0.05)$.

Conclusion RG and AG seem to be effective in enhancing different and sport-specific physical fitness and conditioning. RG enables, indeed, to develop more joints mobility whereas AG improves more strength, balance, and endurance. However, given the small sample size employed, these results should be replicated by further studies utilizing larger samples.
\end{abstract}

Keywords Assessment $\cdot$ Expertise $\cdot$ Gymnastics $\cdot$ High performance $\cdot$ Training process

Luca Russo

info@dottlucarusso.com

1 Department of Clinical, Applied and Biothecnological Sciences, University of L'Aquila, L'Aquila, Italy

2 Department of Public Health, University of Naples "Federico II", Naples, Italy

3 Training Department, Qatar Police College, Doha, Qatar

4 Faculty of Kinesiology, University of Split, Split, Croatia

5 Laboratory for Industrial and Applied Mathematics, Department of Mathematics and Statistics, York University, Toronto, Ontario M3J 1P3, Canada

6 Department of Biomedical Sciences for Health, Università degli Studi di Milano, Milan, Italy

7 Tunisian Research Laboratory "Sport Performance Optimization" National Center of Medicine and Science in Sports, Tunis, Tunisia

\section{Introduction}

The available body of scientific evidence well supports the statement that regularly practicing physical activity provides fundamental health benefits for children and youth [1] especially in the musculoskeletal [2], cardiovascular [3], metabolic [4], immunologic [5], and cognitive [5] domains. According to the World Health Organization (WHO) guidelines, children, and youth aged 5-17 years should perform at least 60 min of moderate to vigorous intensity physical activity daily [1].

Physical activity in childhood was considered to be crucial for the development and acquirement of correct movement patterns in most youth sports- that is to say, those sports where a performance peak is achieved at a relatively young age [6]. Artistic (AG) and rhythmic gymnastics (RG) are age-dependent [7]. Gymnastics training develops, indeed, strength, flexibility, concentration, 
balance, grace, and speed, among others, in young athletes [8]. Both RG and AG are international sports, recognized by the Italian Federation of Gymnastics (FIG). Both these two forms of gymnastics share many similar attributes but differ in terms of events, rules, and style [9]. For females, AG events include performances on different equipment (e.g., vault, uneven bars, balance beam, and floor), while RG events are all performed on the same equipment-a padded floor, that can vary according to the specific support utilized (e.g. rope, hoop, ball, clubs, and ribbon).

It is important that girls and young women participate in sports and develop skills that promote lifelong athletic participation, because of the psychological, sociologic [10], and physiological benefits associated with exercise [11].

Regular trainings in these sports start at the age of 6-7 years. One of the reasons is that the age group ranging from 7 to 11 years old is considered to be a sensitive period for flexibility development [7]. Another reason is probably the intention to start to apply deliberate practice [12] around the age of 10 years old, somewhat before the optimal age for improving power and strength [7]. This age group goes from 12 to 17 years [13] in order to reach peak strength and peak performance of all required skills around the age of 16 [14]. Investing in deliberate practice during childhood and early adolescence becomes vital for achieving the peak performances before adulthood [15]. Within this deliberate practice, physical fitness represents an important factor for success and it provides the basis for the technical skill development. A higher physical fitness level is often related to better performances [16].

Considering the differences of these two sports in terms of events and performances, it is clearly expected that performed movements and demands towards competitors differ as well. Complex tumbling and acrobatics generally characterize women artistic gymnastics. This requires high levels of upper and lower body strength, power, flexibility, and muscular endurance, combined with speed and coordination [17]. Rhythmic gymnastics is mainly characterized by grace presented through the performance of basic acrobatic elements, variety of dance elements and movements, all performed with different props which need to be in constant motion. Generally, rhythmic gymnastics performances require a greater level of flexibility, rhythm, legs strength, endurance, coordination, agility, and balance [18]. In particular, a good compromise between strength and flexibility has been identified as the most contributing factor to performance [19].

Therefore, the aim of this study was to demonstrate how gymnastic training background over the years could modify and develop the physical fitness and to clarify the differences between motor skills achieved by the athletes of artistic and rhythmic gymnastics.

\section{Materials and methods}

\section{Participants}

The present study involved 45 volunteer female subjects, aged between 10 and 12 years, all belonging to the same sports club. The sample was divided into three groups: promotional (PG), rhythmic gymnastics (RG), and artistic gymnastics (AG) group. RG and AG were the case groups, whereas PG acted as control. PG group had 15 subjects (mean age $11.1 \pm 0.9$ years; mean height $141.1 \pm 7.8 \mathrm{~cm}$; mean weight $38.3 \pm 5.4 \mathrm{~kg}$; mean BMI $18.9 \pm 1.8 \mathrm{~kg} / \mathrm{m}^{2}$ ) with a training experience of $5.8 \pm 0.9$ months and $3 \mathrm{~h}$ of training per week. RG group had 15 subjects (mean age $11.1 \pm 1.0$ years; mean height $147.0 \pm 10.5 \mathrm{~cm}$; mean weight $34.3 \pm 6.3 \mathrm{~kg}$; mean BMI $15.7 \pm 0.9 \mathrm{~kg} / \mathrm{m}^{2}$ ) with a training experience of $45.6 \pm 8.0$ months and $9 \mathrm{~h}$ of training per week. AG group had 15 subjects (mean age $11.2 \pm 0.8$ years; mean height $144.9 \pm 9.5 \mathrm{~cm}$; mean weight $36.7 \pm 5.2 \mathrm{~kg}$; mean BMI $17.4 \pm 0.9 \mathrm{~kg} / \mathrm{m}^{2}$ ) with a training experience of $51.5 \pm 7.4$ months and $9 \mathrm{~h}$ of training per week.

The inclusion criteria for the study participations were as follows: (i) absence of any kind of ankle injury or having undergone/undergoing surgery, (ii) no history during the 3 months preceding the test of neuromuscular disease, vertigo, or any uncorrected visual problems, and (iii) no use of sedative medications or analgesic drugs. Subjects not meeting with these criteria were excluded from the study.

Before testing, written informed consent was obtained from the parents/legal guardians of participants (because of their minor age), after they were given a thorough explanation of the purpose, benefits, and potential risks of participating in the study. The protocol conformed to internationally accepted policy statements regarding the use of human participants, in accordance with the Declaration of Helsinki, and was approved by the University's ethics committee.

\section{Findings reporting}

The present study follows the "Strengthening the Reporting of Observational Studies in Epidemiology" (STROBE) Statement guidelines for reporting observational studies [20].

\section{Procedures}

The data collection was carried out through field-based motor tests. Tests were performed in May 2017 in South Italy. All the tests were performed in the gym except for the Coooper's test that was performed on the track and field. 
Data were obtained for (i) joint mobility of the dorsolumbar hinge and the posterior muscular chain by means of the "sit and reach test" (as a measure of the flexibility of the lower back and hamstring muscles), (ii) coxo-femural mobility by means of the "abduction test of the hips" (performed by actively abducting the hips), (iii) scapulo-humeral mobility by means of the "wand test" (as a measure of the flexibility of the scapula-humeral muscles), (iv) balance by means of the "flamingo test" (performed standing on one leg), (v) explosive strength of the lower limbs by means of the "standstill long jump" (performing a long jump starting from standstill), (vi) speed by means of the " $10 \mathrm{~m}$ test" (as a measure of the gait speed), and (vii) endurance by means of the "Cooper's test" (a 12-min run aerobic fitness test, which provides an estimate of $\mathrm{VO}_{2 \max }$ ).

Each athlete was assessed at the same day and the test sequence was the same of the previous list. Each subject respected 3 days of rest between last training session and the test day. All the subjects have been previously instructed on the tests and each subject performed a simulation of each test 2 weeks before the official test day. After 1 week, each test was repeated to assess the reliability of the measures.

\section{Statistical analysis}

The data obtained from the field-based motor tests were processed and calculated as mean and standard deviation (SD). The distribution and presence of abnormal values among the data have been verified before starting any parametric analysis, using the Shapiro-Wilk's test for normality of data distribution. This test was preferred to other tests because of the small sample size employed. Multivariate regressions and generalized linear models were performed to shed light on the determinants of differences between RG and AG groups, utilizing PG as controls. More in details, these analyses were carried out in order to compare different variables and to understand how each physical parameter was influenced by training experience, BMI, age, group, and hours of weekly training. Furthermore, the Pearson coefficient was used to assess correlations between the measured parameters. The statistical correlation tests were performed on the whole sample in order to understand the relationship between different physical skills. Intra-class correlation coefficient (ICC) was used to assess the reliability of the measurements [21]. For each comparison between each group, analysis of variance (ANOVA) was used. Effect sizes (ES) were also computed calculating the Cohen's $d$ (the difference between the means of two groups divided by the pooled standard deviation) [22]. ES up to 0.20 was considered small, up to 0.50 medium, up to 0.80 large, and exceeding 0.80 very large. An a priori sample size power analysis computed a sample of 13 subjects per group to capture a pairwise medium ES with an alpha error probability of 0.05 and a power set at 0.80 . A post hoc sample size power analysis showed that, based on the obtained ES, the recruited sample was adequate, in that ESs were captured with an alpha error probability of 0.05 and a power of 0.95 (a sample in the range of 3-10 subjects per group would have been enough). All statistical analyses were conducted using the commercial software "Statistical Package for Social Sciences" (SPSS, version 23.0.0-IBM Corporation, Armonk, NY, USA). Significance level was set at $p<0.05$.

\section{Results}

Test-retest showed a good reliability (ICC $>0.870$ ). Differences between all measured variables for subsamples of PG, AG, and RG were analyzed at the significance level of $p<0.05$ (Table 1). There are no significant differences for age, height, and weight, but there is a significant difference between subsamples in BMI. Further, a significant difference $(p<0.001)$ was found within the results of the various motor tests and within the years of training. In particular, better values were found in AG, considering the Cooper's endurance test $(1626.7 \pm 7.4 \mathrm{~m})$, the standstill long jump test $(164.1 \pm 11.6 \mathrm{~cm})$ and the Flamingo test $(4.33 \pm 1.35 \mathrm{n} / 60 \mathrm{~s})$. Similarly, RG subsample showed higher values for articular mobility tests, such as coxo-femoral mobility $\left(166.7 \pm 6.3^{\circ}\right)$, sit and reach $(20.5 \pm 1.9 \mathrm{~cm})$, and scapulo-humeral mobility $(45.5 \pm 4.4 \mathrm{~cm})$. In the $10 \mathrm{~m}$ test, $\mathrm{PG}$ subsample showed the worst value, while RG and AG were very similar although RG group showed a better result $(2.30 \pm 0.18 \mathrm{~s})$ with respect to the AG group. Moderate to high significant correlation was found between the motor tests results (Table 2).

The strongest significant correlation was found between the coxo-femoral mobility and the sit and reach test $(r=0.826)$. Results of the multivariate regression are presented in Table 3.

Age seems to significantly impact on the $10 \mathrm{~m}$ speed and the Cooper's tests. The coxo-femoral mobility and the standstill long jump test performance were significantly influenced by the type of training (PG, RG, and AG). Finally, the Cooper's test, the coxo-femural mobility, and the scapulohumeral mobility performance were significantly affected by the hours of weekly training.

\section{Discussion}

The aim of this research was double: the first was to demonstrate how prolonged activity over the years could develop the motor skills of each participant; the second aim was to provide the evidences between two similar sports such as rhythmic and artistic gymnastics. 
Table 1 Sample characteristics broken down according to type of training

\begin{tabular}{|c|c|c|c|c|c|c|c|c|c|c|}
\hline \multirow[t]{2}{*}{ Variables } & \multicolumn{2}{|l|}{ PG } & \multicolumn{2}{|l|}{ RG } & \multicolumn{2}{|l|}{$\mathrm{AG}$} & \multirow[t]{2}{*}{$p$ value } & \multicolumn{3}{|l|}{ ES } \\
\hline & Mean & SD & Mean & SD & Mean & SD & & RG vs PG & AG vs PG & RG vs AG \\
\hline Age (years) & 11.1 & 0.9 & 11.1 & 1.0 & 11.2 & 0.8 & 0.891 & 0.00 & 0.12 & 0.11 \\
\hline Height (cm) & 141.1 & 7.8 & 147.0 & 10.5 & 144.9 & 9.5 & 0.231 & 0.64 & 0.44 & 0.21 \\
\hline Weight (kg) & 38.3 & 5.4 & 34.3 & 6.3 & 36.7 & 5.2 & 0.158 & 0.68 & 0.30 & 0.42 \\
\hline $\operatorname{BMI}\left(\mathrm{kg} / \mathrm{m}^{2}\right)$ & 18.9 & 1.8 & 15.7 & 0.9 & 17.4 & 0.9 & $<0.001$ & 2.25 & 1.05 & 1.89 \\
\hline Hours of training per week & 3.0 & - & 9.0 & - & 9.0 & - & - & - & - & - \\
\hline Training experience (months) & 5.8 & 0.9 & 45.6 & 8.0 & 51.5 & 7.4 & $<0.001$ & 6.99 & 8.67 & 0.77 \\
\hline Cooper test (m) & 1280.7 & 104.0 & 1566.0 & 83.0 & 1626.7 & 74.0 & $<0.001$ & 3.03 & 3.83 & 0.77 \\
\hline Coxo-femoral mobility $\left(^{\circ}\right)$ & 132.1 & 9.0 & 166.7 & 6.3 & 152.1 & 5.2 & $<0.001$ & 4.45 & 2.72 & 2.53 \\
\hline $10 \mathrm{~m}$ speed (s) & 2.57 & 0.15 & 2.30 & 0.18 & 2.35 & 0.13 & $<0.001$ & 1.63 & 1.57 & 0.32 \\
\hline Flamingo test (n/60 s) & 8.53 & 2.20 & 5.07 & 1.49 & 4.33 & 1.35 & $<0.001$ & 1.84 & 2.30 & 0.52 \\
\hline Sit and reach test $(\mathrm{cm})$ & 13.3 & 4.5 & 20.5 & 1.9 & 18.1 & 2.4 & $<0.001$ & 2.08 & 1.33 & 1.11 \\
\hline Standstill long jump (cm) & 125.9 & 15.3 & 135.5 & 7.9 & 164.1 & 11.6 & $<0.001$ & 0.79 & 2.81 & 2.88 \\
\hline Scapulo-humeral mobility (cm) & 67.7 & 5.7 & 45.5 & 4.4 & 51.3 & 8.9 & $<0.001$ & 4.36 & 2.19 & 0.83 \\
\hline
\end{tabular}

$A G$ artistic gymnastics group, $B M I$ body mass index, $E S$ effect size, $P G$ promotional group, $R G$ rhythmic gymnastics group

Table 2 Correlation between the different tests examined

\begin{tabular}{|c|c|c|c|c|c|c|}
\hline & $10 \mathrm{~m}$ speed & Cooper's test & $\begin{array}{l}\text { Coxo-femoral } \\
\text { mobility }\end{array}$ & Flamingo test & Sit and reach test & $\begin{array}{l}\text { Standstill } \\
\text { long Jump }\end{array}$ \\
\hline \multicolumn{7}{|l|}{ Cooper's test } \\
\hline Correlation coefficient & -0.635 & & & & & \\
\hline$p$ value & $<0.001$ & & & & & \\
\hline \multicolumn{7}{|l|}{ Coxo-femoral mobility } \\
\hline Correlation coefficient & -0.540 & 0.687 & & & & \\
\hline$p$ value & 0.001 & $<0.001$ & & & & \\
\hline \multicolumn{7}{|l|}{ Flamingo test } \\
\hline Correlation coefficient & 0.497 & -0.692 & -0.581 & & & \\
\hline$p$ value & 0.005 & $<0.001$ & $<0.001$ & & & \\
\hline \multicolumn{7}{|l|}{ Sit and reach test } \\
\hline Correlation coefficient & -0.512 & 0.621 & 0.826 & -0.469 & & \\
\hline$p$ value & 0.003 & $<0.001$ & $<0.001$ & 0.012 & & \\
\hline \multicolumn{7}{|l|}{ Standstill long jump } \\
\hline Correlation coefficient & -0.441 & 0.639 & 0.257 & -0.542 & 0.270 & \\
\hline$p$ value & 0.024 & $<0.001$ & 0.088 & 0.001 & 0.072 & \\
\hline \multicolumn{7}{|l|}{ Scapulo-humeral mobility } \\
\hline Correlation coefficient & 0.487 & -0.629 & -0.764 & 0.624 & -0.643 & -0.217 \\
\hline$p$ value & 0.007 & $<0.001$ & $<0.001$ & $<0.001$ & 0.001 & 0.152 \\
\hline
\end{tabular}

$A G$ artistic gymnastics group, $B M I$ body mass index, $R G$ rhythmic gymnastics group, $P G$ promotional group

In Cooper test, coxo-femoral mobility, and scapulohumeral mobility, it is possible to observe how hours of training per week are closely related to the results obtained in the motor tests. Those results highlight the purpose of our study and demonstrate that training improves motor abilities. Good motor skills are considered important for children's physical, social, and psychological development and may even be the foundation for an active lifestyle, since several studies have shown a positive association between good motor skills and higher levels of physical activity [23]. Consequently, there is evidence of many health benefits to be gained from an improvement in motor skills [6].

A significant gap is present between the PG and the competitive athletes (RG and $\mathrm{AG}$ ) for the average values of $\mathrm{BMI}$ and all field-based motor tests. Probably, the RG lowest values of BMI are responsible for obtained results; however, 
Table 3 Multivariate regressions and generalized linear models

\begin{tabular}{|c|c|c|c|c|c|}
\hline Independent variables & Coefficient & Standard error & $r_{\text {partial }}$ & $T$ & $p$ \\
\hline \multicolumn{6}{|c|}{$10 \mathrm{~m}$ speed $(\mathrm{s}): R^{2}=0.71$, adapted $R^{2}=0.67$} \\
\hline (Constant) & 4.000 & & & & \\
\hline Training experience (months) & -0.003 & 0.003 & -0.154 & -0.975 & 0.335 \\
\hline BMI & -0.007 & 0.014 & -0.084 & -0.527 & 0.601 \\
\hline Age (years) & -0.111 & 0.023 & -0.614 & -4.854 & $<0.001$ \\
\hline Type of training & 0.093 & 0.048 & 0.297 & 1.939 & 0.059 \\
\hline Hours of weekly training & -0.044 & 0.027 & -0.255 & -1.646 & 0.107 \\
\hline \multicolumn{6}{|c|}{ Cooper's test $(\mathrm{m}): R^{2}=0.81$, adapted $R^{2}=0.79$} \\
\hline (Constant) & 285.184 & & & & \\
\hline Training experience (months) & -3.519 & 2.382 & -0.230 & -1.477 & 0.147 \\
\hline BMI & 10.159 & 10.114 & 0.158 & 1.004 & 0.321 \\
\hline Age (years) & 51.147 & 16.747 & 0.439 & 3.054 & 0.004 \\
\hline Type of training & 57.140 & 35.184 & 0.251 & 1.624 & 0.112 \\
\hline Hours of weekly training & 66.836 & 19.506 & 0.481 & 3.426 & 0.001 \\
\hline \multicolumn{6}{|c|}{ Coxo-femoral mobility $\left({ }^{\circ}\right): R^{2}=0.84$, adapted $R^{2}=0.82$} \\
\hline (Constant) & 110.945 & & & & \\
\hline Training experience (months) & 0.029 & 0.201 & 0.0234 & 0.146 & 0.885 \\
\hline BMI & 1.583 & 0.852 & 0.285 & 1.857 & 0.071 \\
\hline Age (years) & -1.775 & 1.412 & -0.197 & -1.257 & 0.216 \\
\hline Type of training & -17.305 & 2.965 & -0.683 & -5.836 & $<0.001$ \\
\hline Hours of weekly training & 9.318 & 1.644 & 0.672 & 5.668 & $<0.001$ \\
\hline \multicolumn{6}{|c|}{ Flamingo test (times): $R^{2}=0.60$, adapted $R^{2}=0.54$} \\
\hline (Constant) & 1.808 & & & & \\
\hline Training experience (months) & -0.069 & 0.050 & -0.218 & -1.396 & 0.171 \\
\hline BMI & 0.372 & 0.210 & 0.272 & 1.767 & 0.085 \\
\hline Age (years) & 0.030 & 0.348 & 0.014 & 0.087 & 0.931 \\
\hline Type of training & -0.966 & 0.732 & -0.207 & -1.321 & 0.194 \\
\hline Hours of weekly training & 0.242 & 0.406 & 0.095 & 0.597 & 0.554 \\
\hline \multicolumn{6}{|c|}{ Sit and reach test $(\mathrm{cm}): R^{2}=0.49$, adapted $R^{2}=0.43$} \\
\hline (Constant) & 12.139 & & & & \\
\hline Training experience (months) & 0.036 & 0.096 & 0.059 & 0.369 & 0.714 \\
\hline BMI & 0.163 & 0.405 & 0.064 & 0.401 & 0.690 \\
\hline Age (years) & -0.343 & 0.671 & -0.082 & -0.511 & 0.612 \\
\hline Type of training & -2.839 & 1.410 & -0.307 & -2.014 & 0.051 \\
\hline Hours of weekly training & 1.516 & 0.782 & 0.297 & 1.939 & 0.060 \\
\hline \multicolumn{6}{|c|}{ Standstill long jump: $R^{2}=0.74$, adapted $R^{2}=0.71$} \\
\hline (Constant) & 28.067 & & & & \\
\hline Training experience (months) & 0.288 & 0.321 & 0.142 & 0.896 & 0.376 \\
\hline BMI & 2.198 & 1.362 & 0.250 & 1.613 & 0.115 \\
\hline Age (years) & 3.675 & 2.256 & 0.252 & 1.629 & 0.111 \\
\hline Type of training & 22.670 & 4.739 & 0.608 & 4.784 & $<0.001$ \\
\hline Hours of weekly training & -2.905 & 2.627 & -0.174 & -1.106 & 0.276 \\
\hline \multicolumn{6}{|c|}{ Scapulo-humeral mobility $(\mathrm{cm}): R^{2}=0.71$, adapted $R^{2}=0.67$} \\
\hline (Constant) & 39.208 & & & & \\
\hline Training experience (months) & -0.052 & 0.194 & -0.043 & -0.269 & 0.789 \\
\hline BMI & 0.902 & 0.822 & 0.173 & 1.097 & 0.279 \\
\hline Age (years) & 1.636 & 1.360 & 0.189 & 1.203 & 0.236 \\
\hline Type of training & 4.415 & 2.858 & 0.240 & 1.545 & 0.131 \\
\hline Hours of weekly training & -3.605 & 1.584 & -0.342 & -2.276 & 0.028 \\
\hline
\end{tabular}

$A G$ artistic gymnastics group, $B M I$ body mass index, $R G$ rhythmic gymnastics group, $P G$ promotional group 
this needs to be confirmed in other analysis. Differences are present also for RG and AG because of the intrinsic difference of sport-specific stimulus and relative adaptations. RG athletes seem to be better than AG athletes in lower limb mobility tasks such as coxo-femural mobility and sit and reach test, while AG athletes are better than RG athletes in endurance, strength and power task, balance, and upper limb mobility. Only the speed seems to be very similar with only $0.05 \mathrm{~s}$ of difference in favour of the RG athletes.

These differences strictly depend on the physical adaptation of the athletes to the sport-specific training load. In fact, the artistic gymnastics aim is to develop exercises on the ground for competition lasting 1'30" with diagonals made of continuous technical elements, which require aerobic resistance. In the rhythmic group, instead, the competition exercises are less dynamic, with considerable changes of rhythm [24]. The balance performance, as well as the endurance, is better in AG athletes because of the similarity of the Flamingo test with sport-specific movements of artistic gymnastics. In fact, during the training on the beam, the AG athletes perform jumps and evolution on a surface $10 \mathrm{~cm}$ wide, unlike the RG athletes, who work on the entire surface of the ground [25]. Moreover, the training of AG athletes provides a higher stimulus on strength and power with respect to the RG athletes training. In artistic gymnastics, there are various evolutions in flight and in a single jump with different rotations on the different body axes [25]; this can explain the higher performance in standstill long jump for the AG with respect to the RG. Sport-specific adaptations are present also for the RG. In fact, this group showed better values for lower limb and trunk mobility. This kind of training is fundamental in rhythmic gymnastics because it allows reaching high degrees of articular excursion, which is used to perform positions of fluency and fundamental elements in the composition of the competition exercises.

The data fundamentally depend on the type of training: in fact, the RG works more on joint mobility and muscle lengthening while the $\mathrm{AG}$ works on strength and power to perform specific technical gestures in difficult balance conditions. This is in line with results of Vicente-Rodriguez et al. [26] that found recreational artistic gymnastic participation is associated with delayed pubertal development, enhanced physical fitness, muscle mass, and bone density in prepubertal girls, eliciting a higher osteogenic stimulus than rhythmic gymnastics. The deterioration of growth potential in female artistic gymnastic is also observed in Georgopoulos et al. [27]. According to these results, the training of artistic gymnastics should be based on exercises of both strength and endurance organized gradually by intensity, speed of execution, and number of repetitions and series, which affect the muscular districts in a balanced manner, left and right part, and upper and lower part of the body. Instead, the training of rhythmic gymnastics should be based on stretching and mobility according to the technical elements of the competition exercises, in order to achieve the best specific strength. One of the principal limitations of this study is the difference in terms of months of training expertise between the PG, $\mathrm{RG}$, and AG groups. Although this value is not related to any performance in motor test, according to the multivariate regression, it should be better to compare in future studies some PG with the same time of training experience with respect to the competitive groups.

Finally, the data of this study confirm the previous literature on the centrality to train in a specific way in order to achieve a good physical condition; furthermore, it is clear the difference between the performances among the motor tests of the PG with respect to the two competitive groups RG and AG. The significant relationship between the amounts of hours of weekly training and the performance of some motor tests is a strong index of the necessity to improve physical training in order to achieve better physical results. In conclusion, we estimated the difference between the results achieved by the athletes of AG and RG highlighting strength, endurance, and balance more in the AG, and the articular mobility more in the RG.

\section{Conclusions}

In conclusion, rhythmic and artistic gymnastics seem to be effective in enhancing different and sport-related physical skills and adaptations. Rhythmic gymnastics develops more joints mobility and artistic gymnastics develops more strength, balance, and endurance. Present results should be implemented by other studies involving bigger samples.

Acknowledgements The authors would like to acknowledge a highly motivated group of subjects that made this investigation possible.

Funding Open access funding provided by Università degli Studi dell'Aquila within the CRUI-CARE Agreement.

\section{Compliance with ethical standards}

Conflict of interest The authors declare that they have no conflict of interest.

Ethical approval The protocol conformed to internationally accepted policy statements regarding the use of human participants, in accordance with the Declaration of Helsinki, and ethical approval was agreed by the ethical committee of the institution Novi Sad University.

Informed consent Informed consent was obtained from all the parents/ legal guardians of participants (because of their minor age), after they were given a thorough explanation of the purpose, benefits, and potential risks of participating in the study. 
Open Access This article is licensed under a Creative Commons Attribution 4.0 International License, which permits use, sharing, adaptation, distribution and reproduction in any medium or format, as long as you give appropriate credit to the original author(s) and the source, provide a link to the Creative Commons licence, and indicate if changes were made. The images or other third party material in this article are included in the article's Creative Commons licence, unless indicated otherwise in a credit line to the material. If material is not included in the article's Creative Commons licence and your intended use is not permitted by statutory regulation or exceeds the permitted use, you will need to obtain permission directly from the copyright holder. To view a copy of this licence, visit http://creativecommons.org/licenses/by/4.0/.

\section{References}

1. World Health Organization-WHO (2015) Physical activity and young people. https://www.who.int/dietphysicalactivity/facts heet_young_people/en/. Accessed 01 Apr 2020

2. Smith JJ, Eather N, Morgan PJ, Plotnikoff RC, Faigenbaum AD, Lubans DR (2014) The health benefits of muscular fitness for children and adolescents: a systematic review and meta-analysis. Sport Med 44:1209-1223

3. Santos DA, Marques A, Minderico CS, Ekelund U, Sardinha LB (2018) A cross-sectional and prospective analyse of reallocating sedentary time to physical activity on children's cardiorespiratory fitness. J Sports Sci 36:1720-1726

4. Schmitz K, Jacobs D, Hong CP, Steinberger J, Moran A, Sinaiko A (2002) Association of physical activity with insulin sensitivity in children. Int J Obes 26:1310-1316

5. Sirico F, Bianco A, D'Alicandro G, Castaldo C, Montagnani S, Spera R, Nurzynska D (2018) Effects of physical exercise on Adiponectin, leptin, and inflammatory markers in childhood obesity: systematic review and meta-analysis. Child Obes 14:207-217

6. Hestbaek L, Andersen ST, Skovgaard T, Olesen LG, Elmose M, Bleses D et al (2017) Influence of motor skills training on children's development evaluated in the motor skills in PreSchool (MiPS) study-DK: study protocol for a randomized controlled trial, nested in a cohort study. Trials 18:400

7. Malina RM, Baxter-Jones ADG, Armstrong N, Beunen GP, Caine D, Daly RM, Lewis RD, Rogol AD, Russell K (2013) Role of intensive training in the growth and maturation of artistic gymnasts. Sports Med 43:783-802

8. Zetaruk MN (2000) The young gymnast. Clin Sports Med 19:757-780

9. Root H, Marshall AN, Thatcher A, Valier ARS, Valovich McLeod TC, Bay RC (2019) Sport specialization and fitness and functional task performance among youth competitive gymnasts. J Athl Train 54:1095-1104

10. Padulo J, Bragazzi NL, De Giorgio A, Grgantov Z, Prato S, Ardigò LP (2019) The effect of physical activity on cognitive performance in an Italian elementary school: insights from a pilot study using structural equation modeling. Front Physiol 10:202

11. Van de Loo DA, Johnson MD (1995) The young female athlete. Clin Sports Med 14:687-707

12. Cliff DP, Okely AD, Burrows TL, Jones RA, Morgan PJ, Collins CE, A Buar L (2013) Objectively measured sedentary behavior, physical activity, and plasma lipids in overweight and obese children. Obesity 21:382-385

13. Josie Gleave A, Cole-Hamilton I (2019) A world without play: a literature review on the effects of a lack of play on children's lives [Internet]. https://www.playengland.org.uk. Accessed $01 \mathrm{Apr}$ 2020

14. Arkaev LI, Suchilin NG (2004) How to create champions: the theory and methodology of training top-class gymnasts. Meyer $\&$ Meyer Sport, Auflage

15. Côté J, Gilbert W (2009) An integrative definition of coaching effectiveness and expertise. Int J Sports Sci Coach 4:307-323

16. Tran TT, Lundgren L, Secomb J, Farley ORL, Haff GG, Seitz LB, Sheppard JM (2015) Comparison of physical capacities between nonselected and selected elite male competitive surfers for the National Junior Team. Int J Sports Physiol Perform 10:178-182

17. Jemni M, Sands WA, Friemel F, Stone MH, Cooke CB (2006) Any effect of gymnastics training on upper-body and lower-body aerobic and power components in national and international male gymnasts? J Strength Cond Res 20:899-907

18. Stathokostas L, Little RMD, Vandervoort AA, Paterson DH (2012) Flexibility training and functional ability in older adults: a systematic review. J Aging Res 2012:306818. https://doi. org/10.1155/2012/306818

19. Donti O, Tsolakis C, Bogdanis GC (2014) Effects of baseline levels of flexibility and vertical jump ability on performance following different volumes of static stretching and potentiating exercises in elite gymnasts. J Sports Sci Med 13:105-113

20. Vandenbroucke JP, von Elm E, Altman DG, Gøtzsche PC, Mulrow CD, Pocock SJ, Poole C, Schlesselman JJ, Egger M (2007) Strengthening the reporting of observational studies in epidemiology (STROBE): explanation and elaboration. PLoS Med 4:e297

21. Hopkins WG (2000) Measures of reliability in sports medicine and science. Sports Med 30:1-15

22. Cohen J (1988) Statistical power analysis for the behavioral sciences, 2nd edn. Academic Press, New York

23. Lubans Lubans DR, Morgan PJ, Cliff DP, Barnett LM, Okely AD (2010) Fundamental movement skills in children and adolescents. Sport Med 40:1019-1035

24. Vladimir P, Marian C, Olivia T (2013) Learning and transfer in Women's artistic gymnastics. Proc Soc Behav Sci 93:23-28

25. Morgan E (2017) Gymnastics: science on the mat and in the air. Greenhaven Publishing LLC, New York, p 108. https://www. worldcat.org/title/gymnastics-science-on-the-mat-and-in-the-air/ oclc/1041042022. Accessed 01 Apr 2020

26. Vicente-Rodriguez G, Dorado C, Ara I, Perez-Gomez J, Olmedillas H, Delgado-Guerra S, Calbet JAL (2007) Artistic versus rhythmic gymnastics: effects on bone and muscle mass in young girls. Int J Sports Med 28:386-393

27. Georgopoulos NA, Markou KB, Theodoropoulou A, Benardot D, Leglise M, Vagenakis AG (2002) Growth retardation in artistic compared with rhythmic elite female gymnasts. J Clin Endocrinol Metab 87:3169-3173

Publisher's Note Springer Nature remains neutral with regard to jurisdictional claims in published maps and institutional affiliations. 